TOKYO J. MATH.

VoL. 32, No. 2, 2009

\title{
Scattering for the Klein-Gordon Equations with Time-Dependent Potentials
}

\author{
Sojiro MURAI
}

Tokai University

(Communicated by H. Ohta)

\begin{abstract}
The present paper is to investigate the existence of the scattering operators for the Klein-Gordon equations with a potential which depends on both the space and time variables, and admits a singularity in $L^{q}\left(\mathbf{R}^{n}\right)$ sense. The main tool is the Strichartz estimates of solutions.
\end{abstract}

\section{Introduction and Result} tential:

This paper is devoted to the scattering theory for the Klein-Gordon equation with a po-

$$
u_{t t}-\Delta u+m^{2} u+V(x, t) u=0, \quad(x, t) \in \mathbf{R}^{n} \times \mathbf{R},
$$

where $u_{t t}=\partial^{2} u / \partial t^{2}, \Delta$ is the $n$-dimensional Laplacian, $n \geq 1, m$ is a positive constant and $V(x, t)$ is a real-valued potential. $E$ is the space of all pairs $\vec{f}={ }^{t}\left(f_{1}, f_{2}\right)$ of functions such that

$$
\|\vec{f}\|_{E}^{2}=\frac{1}{2} \int_{\mathbf{R}^{n}}\left\{\left|\nabla f_{1}(x)\right|^{2}+m^{2} f_{1}(x)^{2}+f_{2}(x)^{2}\right\} d x<\infty
$$

this norm is called the energy.

As an evolution equation in $E$, the perturbed problem (1.1) with the initial data $\vec{f}=$ ${ }^{t}\left(f_{1}, f_{2}\right) \in E$ is rewritten in the matrix form

$$
i \partial_{t} \vec{u}=\Lambda_{0} \vec{u}+\vec{V}(t) \vec{u},
$$

where $i=\sqrt{-1}, \vec{u}(t)={ }^{t}\left(u(t), u_{t}(t)\right)$,

$$
\Lambda_{0}=i\left(\begin{array}{cc}
0 & 1 \\
\Delta-m^{2} & 0
\end{array}\right), \quad \vec{V}(t)=-i\left(\begin{array}{cc}
0 & 0 \\
V(x, t) & 0
\end{array}\right) .
$$

Let $U_{0}(t), t \in \mathbf{R}$, be the unitary group in $E$ which represents the solution of the free equation

$$
i \partial_{t} \vec{u}_{0}=\Lambda_{0} \vec{u}_{0}
$$

Received June 24, 2008; revised February 24, 2009 
and is of the form

$$
U_{0}(t)=\left(\begin{array}{ll}
\dot{K}(t) & K(t) \\
\ddot{K}(t) & \dot{K}(t)
\end{array}\right),
$$

where $K(t)=A^{-1} \sin (t A), A=\left(m^{2}-\Delta\right)^{1 / 2}$. By using the operator $U_{0}(t)$, the problem (1.2) is reduced to the integral equation

$$
\vec{u}(t)=U_{0}(t) \vec{f}-i \int_{0}^{t} U_{0}(t-\tau) \vec{V}(\tau) \vec{u}(\tau) d \tau .
$$

It will be proved in Proposition 2.3 that, for given $\vec{f} \in E$, (1.4) has a unique solution $\vec{u}(t) \in$ $C(\mathbf{R} ; E)$, if $V(x, t)$ is small in the space $L^{v}\left(\mathbf{R}_{t} ; L^{q}\left(\mathbf{R}_{x}^{n}\right)\right)$ for some pair $(q, v)$. We denote by $U(t, s) \in \mathcal{B}(E)$ (the space of all bounded linear operators on $E$ ) the evolution operator which maps the solutions at time $s$ to those at time $t$ :

$$
\vec{u}(t)=U(t, s) \vec{u}(s) .
$$

The unique existence of the solutions of (1.4) implies that, for any fixed $s$ and $t, U(t, s)$ defines a bijection on $E$. In the present paper we write $\int_{\mathbf{R}^{n}}=\int$ for brevity. We define also the energy of the solutions of (1.1) at time $t$ :

$$
\|\vec{u}(t)\|_{E}^{2}=\frac{1}{2} \int\left\{\left|u_{t}(x, t)\right|^{2}+|\nabla u(x, t)|^{2}+m^{2}|u(x, t)|^{2}\right\} d x,
$$

where $\nabla u$ is the gradient of $u$.

If $V$ is small in $C^{1}(\mathbf{R} ; X)$ with a suitable Banach space $X$ and the coefficient $m=$ $m(x)$ decays in the space variable $x$, then it is shown in [11] that the scattering operators are constructed on the basis of the weighted energy method due to $[9,10]$ which treated, in fact, the energy decay-nondecay problems for the dissipative wave equations. But then, compared with [11], it is rather natural in physical criterion that the mass $m$ is constant and $V(x, t)$ has a singularity in some point $x_{0} \in \mathbf{R}^{n}$. However, the method of $[9,10,11]$ is not effective in the present case. Our strategy is forced to be different from $[9,10,11]$, say, to use the appropriate Strichartz estimates for (1.1) (see Lemma 2.1 below) which can be obtained by the crucial results of Hayashi and Naumkin [2] for the potentially free Klein-Gordon equations (see also D'Ancona and Fanelli [1] for the end point Strichartz estimates). On the basis of these estimates the scattering operators will be constructed (see Theorem 1.1).

In contrast with the novelty of our problem, we mention the related problems with timedependent potentials. For example, Mochizuki and Motai constructed the scattering operators for the Schrödinger equations with time-dependent complex potentials under the smallness conditions on the potential (see [8]). Their method is based on the Strichartz estimates for the free and the perturbed solutions. However, as far as we know, there is no result on the scattering problems for the Klein-Gordon equations with time-dependent potentials. Note that time-dependent real potentials have been treated in Howland [3], Kitada and Yajima [6], Yafaev [12] and Yajima [13] without requiring any smallness condition on the potentials. For 
the time independent complex potentials, the so called smooth perturbation theory has been developed in Kato's classical paper [4] (see also [5]). Repeatedly speaking, our potential $V$ depends on $x$ and $t$; thus the theory of [4] could not be applied to our case.

In order to state our result, we make the following assumption:

Assumption A. $V(x, t)$ belongs to $L^{v}\left(\mathbf{R} ; L^{q}\right)$, where

$$
0 \leq \frac{1}{q} \leq \frac{2}{n+2}, \quad \frac{1}{v}=1-\frac{n}{2 q}
$$

and is assumed to be the following smallness condition:

$$
C_{1}\|V\|_{L^{v}\left(\mathbf{R} ; L^{q}\right)}<1
$$

with a certain constant $C_{1}=C_{1}(n, q)$ given in Lemma 2.1 below.

For the convenience of the readers, let us introduce some examples satisfying Assumption A.

EXAMPLES. The functions $V(x, t)$ satisfying Assumption A are as follows:

(i) $V(x, t)=V_{0}(1+r)^{-\alpha}(1+t)^{-\beta}, \quad \alpha>\frac{n}{q}, \quad \beta>\frac{1}{v}$,

with a small constant $V_{0} \in \mathbf{R}$.

(ii) Let $\chi_{j}(x) \in C_{0}^{\infty}\left(\mathbf{R}^{n}\right)$ be equal to 1 in a small neighborhood of $x_{j}(j=1, \ldots, N)$, and 0 outside of a neighborhood of $x_{j}$, and let $V_{0} \in \mathbf{R}$ be a small constant. We put

$$
V(x, t)=V_{0}\left\{\sum_{j=1}^{N} \chi_{j}(x)\left|x-x_{j}\right|^{-\alpha_{j}}+\sum_{j=1}^{N}\left(1-\chi_{j}(x)\right) w(x)\right\}(1+t)^{-\beta},
$$

where $\alpha_{j}<\frac{n}{q}(j=1, \ldots, N), \beta>\frac{1}{v}$ and $w(x) \in L^{q}\left(\mathbf{R}^{n}\right)$.

We shall prove here the following:

THEOREM 1.1. Let $n \geq 1$ and suppose Assumption A. Then the following assertions hold:

(i) For every $\vec{f} \in E$ there exists $\vec{f}_{0}^{ \pm} \in E$ such that

$$
\left\|U(t, 0) \vec{f}-U_{0}(t) \vec{f}_{0}^{ \pm}\right\|_{E} \rightarrow 0 \quad \text { as } \quad t \rightarrow \pm \infty .
$$

(ii) Put

$$
Z^{ \pm}=s-\lim _{t \rightarrow \pm \infty} U_{0}(-t) U(t, 0) .
$$

Then $Z^{ \pm}$defines a nontrivial bounded operator on $E$.

(iii) If we restrict (1.6) in Assumption A to

$$
\left(C_{1}+C_{2}\right)\|V\|_{L^{v}\left(\mathbf{R} ; L^{q}\right)}<1,
$$


with a certain constant $C_{2}=C_{2}(n, q, m)$ given in Lemma 2.2 below, then $Z^{ \pm}$gives a bijection on E. Thus, the scattering operator

$$
S=Z^{+}\left(Z^{-}\right)^{-1}: \vec{f}_{0}^{-} \rightarrow \vec{f}_{0}^{+}
$$

is well-defined and also gives a bijection on $E$.

This paper is organized as follows: In section 2 we will give the Strichartz estimates which play an important role to prove our theorem. Finally in section 3 we prove Theorem 1.1 .

\section{Strichartz estimates}

In this section, we shall derive some Strichartz estimates for (1.2)-(1.3). Let us first introduce the Strichartz estimates for the free solutions due to D'Ancona and Fanelli [1] and Hayashi and Naumkin [2], and then use it to obtain similar estimates for the perturbed solutions. In the sequel we often use the following notation: $H_{p}^{k}=H_{p}^{k}\left(\mathbf{R}^{n}\right)$ is the usual Sobolev space for $1 \leq p \leq \infty$ and $k \in \mathbf{R}$ with norm

$$
\|u\|_{H_{p}^{k}}=\left\|\mathcal{F}^{-1}\left(\langle\xi\rangle^{k} \hat{u}(\xi)\right)\right\|_{L^{p}},
$$

where $\hat{u}(\xi)$ denotes the Fourier transformation of $u(x), \mathcal{F}^{-1}$ is its inverse and $\langle\xi\rangle=(1+$ $\left.|\xi|^{2}\right)^{1 / 2}$. We also write $H^{k}=H_{2}^{k}$. Denote the space-time norm of $\phi$ in $L^{r}\left(I ; L^{q}\right)$ by

$$
\|\phi\|_{L^{r}\left(I ; L^{q}\right)}=\|\| \phi(t)\left\|_{L^{q}\left(\mathbf{R}^{n}\right)}\right\|_{L^{r}(I)},
$$

where $I$ is a bounded or unbounded time interval. The following lemma is proved in D'Ancona and Fanelli [1] and Hayashi and Naumkin [2].

Lemma $2.1([1,2])$. Let $n \geq 1, \frac{n-2}{2 n} \leq \frac{1}{p} \leq \frac{1}{2}$ and $\frac{1}{r}=\frac{n}{2}\left(\frac{1}{2}-\frac{1}{p}\right)$. Then there exists a constant $C_{1}=C_{1}(n, p)>0$ such that

$$
\begin{aligned}
\left\|\int_{s}^{t} e^{i(t-\tau) A} g(\tau) d \tau\right\|_{L^{r}\left(I ; L^{p}\right)} & \leq C_{1}\|g\|_{L^{r^{\prime}\left(I ; H_{p^{\prime}}\right)}}, \\
\left\|\int_{S}^{t} e^{i(t-\tau) A} g(\tau) d \tau\right\|_{L^{\infty}\left(I ; L^{2}\right)} & \leq C_{1}^{1 / 2}\|g\|_{L^{r^{\prime}\left(I ; H_{p^{\prime}}^{\mu}\right)}},
\end{aligned}
$$

and

$$
\left\|e^{i t A} \phi\right\|_{L^{r}\left(I ; L^{p}\right)} \leq C_{1}^{1 / 2}\|\phi\|_{H^{\mu}},
$$

where $\frac{1}{r}+\frac{1}{r^{\prime}}=1, \frac{1}{p}+\frac{1}{p^{\prime}}=1$ and $\mu=\left(1+\frac{n}{2}\right)\left(\frac{1}{2}-\frac{1}{p}\right)$.

By using the estimate (2.3) from Lemma 2.1, we have the following estimates for the free solution $u_{0}(t)$ of (1.3). 
LeMma 2.2. Let $n, p, r$ and $\mu$ be as in Lemma 2.1. Then the solution $\vec{u}_{0}(t)=$ ${ }^{t}\left(u_{0}(t), u_{0 t}(t)\right)$ of $(1.3)$ with the data $\vec{f}_{0}={ }^{t}\left(f_{01}, f_{02}\right) \in E$ satisfies the following estimates:

$$
\begin{aligned}
\left\|u_{0}\right\|_{L^{r}\left(I ; H_{p}^{1-\mu}\right)} \leq\left(2 C_{2}\right)^{1 / 2}\left\|\vec{f}_{0}\right\|_{E}, \\
\left\|u_{0 t}\right\|_{L^{r}\left(I ; H_{p}^{-\mu}\right)} \leq\left(2 C_{2}\right)^{1 / 2}\left\|\vec{f}_{0}\right\|_{E} .
\end{aligned}
$$

PROOF. We can write $\vec{u}_{0}(t)$ as

$$
\vec{u}_{0}(t)=\left(\begin{array}{cc}
\dot{K}(t) & K(t) \\
\ddot{K}(t) & \dot{K}(t)
\end{array}\right) \vec{f}_{0}, \quad K(t)=A^{-1} \sin (t A) .
$$

We can estimate, by using (2.3),

$$
\begin{aligned}
\left\|u_{0}\right\|_{L^{r}\left(I ; H_{p}^{1-\mu}\right)} & \leq\left\|\cos (t A) f_{01}\right\|_{L^{r}\left(I ; H_{p}^{1-\mu}\right)}+\left\|A^{-1} \sin (t A) f_{02}\right\|_{L^{r}\left(I ; H_{p}^{1-\mu}\right)} \\
& \leq C_{1}^{1 / 2}\left(\left\|f_{01}\right\|_{H^{1}}+\left\|f_{02}\right\|_{L^{2}}\right) \\
& \leq\left(2 C_{1}\right)^{1 / 2}\left(\left\|f_{01}\right\|_{H^{1}}^{2}+\left\|f_{02}\right\|_{L^{2}}^{2}\right)^{1 / 2} \\
& \leq\left(2 C_{2}\right)^{1 / 2}\left\|\vec{f}_{0}\right\|_{E}
\end{aligned}
$$

This proves (2.4). In the same way, we have the required estimate (2.5):

$$
\begin{aligned}
\left\|u_{0 t}\right\|_{L^{r}\left(I ; H_{p}^{-\mu}\right)} & \leq\left\|A \sin (t A) f_{01}\right\|_{L^{r}\left(I ; H_{p}^{-\mu}\right)}+\left\|\cos (t A) f_{02}\right\|_{L^{r}\left(I ; H_{p}^{-\mu}\right)} \\
& \leq C_{1}^{1 / 2}\left(\left\|f_{01}\right\|_{H^{1}}+\left\|f_{02}\right\|_{L^{2}}\right) \\
& \leq\left(2 C_{1}\right)^{1 / 2}\left(\left\|f_{01}\right\|_{H^{1}}^{2}+\left\|f_{02}\right\|_{L^{2}}^{2}\right)^{1 / 2} \\
& \leq\left(2 C_{2}\right)^{1 / 2}\left\|\vec{f}_{0}\right\|_{E} .
\end{aligned}
$$

This ends the proof of Lemma 2.2.

For $1 \leq \gamma \leq \infty$ and $\pm s \geq 0$, we put

$$
L_{ \pm, s}^{\gamma} X=L^{\gamma}\left(\mathbf{R}_{ \pm, s} ; X\right)
$$

where $\mathbf{R}_{+, s}=[s, \infty)$ for $s \geq 0, \mathbf{R}_{-, s}=(-\infty, s]$ for $s \leq 0$ and $X$ is a Banach space. In case of $s=0$, we simply write $\mathbf{R}_{ \pm, 0}=\mathbf{R}_{ \pm}$. By Assumption A we have $V(x, t) \in L_{ \pm, s}^{v} L^{q}$ for any $\pm s \geq 0$. Moreover, we see from (1.6) that the following inequality

$$
C_{1}\|V\|_{L_{ \pm, s}^{v} L^{q}}<1
$$

holds for any $\pm s \geq 0$. In the following we restrict the pair $(p, r)$ in Lemma 2.1 as follows:

$$
\frac{n}{2(n+2)} \leq \frac{1}{p} \leq \frac{1}{2}, \quad \frac{1}{r}=\frac{n}{2}\left(\frac{1}{2}-\frac{1}{p}\right) .
$$


For this exponent $p$, it can be checked that the condition on $\mu$ in Lemma 2.1 is to be $\mu \leq \frac{1}{2}$. Moreover, we choose the pair $(p, r)$ in (2.7), related to $(q, v)$, as follows:

$$
\frac{1}{p}=\frac{1}{2}\left(1-\frac{1}{q}\right), \quad \frac{1}{r}=\frac{1}{2}\left(1-\frac{1}{v}\right) .
$$

As is easily seen, the condition for $(q, v)$ in Assumption A is equivalent to that for $(p, r)$ in (2.7). Then we have the following:

Proposition 2.3. Let $n \geq 1$ and suppose Assumption A. Let $s \in \mathbf{R}$ be arbitrarily fixed. Then for each $\vec{f}={ }^{t}\left(f_{1}, f_{2}\right) \in E$, the following assertions hold:

(i) The integral equation

$$
u(t)=\dot{K}(t-s) f_{1}+K(t-s) f_{2}-\int_{s}^{t} K(t-\tau) V(\tau) u(\tau) d \tau
$$

has a unique solution $u(t) \in L_{ \pm, s}^{r} H_{p}^{1-\mu}$.

(ii) The solution $u(t)$ of (2.9) belongs to $C\left(\mathbf{R}_{ \pm, s} ; H^{1}\right)$ and coincides with $U(t, s) \vec{f}$. Moreover, we have

$$
\begin{gathered}
\|u\|_{L_{ \pm, s}^{r} H_{p}^{1-\mu}} \leq \frac{\left(2 C_{2}\right)^{1 / 2}}{1-C_{1}\|V\|_{L_{ \pm s}^{v} L^{q}}}\|\vec{f}\|_{E}, \\
\left\|\int_{s}^{t} K(t-\tau) V(\tau) u(\tau) d \tau\right\|_{H^{1}} \leq \frac{\sqrt{2 C_{1} C_{2}}\|V\|_{L_{ \pm s s}^{v} L^{q}}}{1-C_{1}\|V\|_{L_{ \pm, s}^{v} L^{q}}}\|\vec{f}\|_{E} .
\end{gathered}
$$

ProOF. (i) For $g=g(x, t) \in L_{ \pm, s}^{r} H_{p}^{1-\mu}$, we put

$$
\Phi_{ \pm, s}[g](t)=-\int_{s}^{t} K(t-\tau) V(\tau) g(\tau) d \tau, \quad t \in \mathbf{R}_{ \pm} .
$$

Then it can be checked that

$$
\left\|\Phi_{ \pm, s}[g]\right\|_{L_{ \pm, s}^{r} H_{p}^{1-\mu}} \leq C_{1}\|V\|_{L_{ \pm, s}^{v}}{ }^{q}\|g\|_{L_{ \pm, s}^{r} H_{p}^{1-\mu}} .
$$

Indeed, by the estimate (2.1) we have

$$
\left\|\int_{s}^{t} K(t-\tau) V(\tau) g(\tau) d \tau\right\|_{L_{ \pm, s}^{r} H_{p}^{1-\mu}} \leq C_{1}\|V g\|_{L_{ \pm, s}^{r^{\prime}} H_{p^{\prime}}^{\mu}} \leq C_{1}\|V g\|_{L_{ \pm, s}^{r^{\prime}} H_{p^{\prime}}^{1-\mu}},
$$

since $\mu \leq \frac{1}{2}$. Here, by using Hölder's inequality, we see

$$
\|V g\|_{L_{ \pm, s}^{r^{\prime}} H_{p^{\prime}}^{1-\mu}} \leq\|V\|_{L_{ \pm, s}^{v} L^{q}}\|g\|_{L_{ \pm, s}^{r^{\prime} v /\left(v-r^{\prime}\right)} H_{p^{\prime} q /\left(q-p^{\prime}\right)}^{1-\mu}} .
$$

Since (2.8) implies $\frac{p^{\prime} q}{q-p^{\prime}}=p$ and $\frac{r^{\prime} v}{v-r^{\prime}}=r$, the estimate (2.14) together with (2.13) imply (2.12). As a consequence of (2.12), we have also

$$
\left\|\Phi_{ \pm, s}\right\|_{\mathcal{B}\left(L_{ \pm, s}^{r} H_{p}^{1-\mu}\right)} \leq C_{1}\|V\|_{L_{ \pm, s}^{v} L^{q}} .
$$


Now, going back to the integral equation (2.9), we consider the solution $u(t)$ of (2.9). For $\vec{f}={ }^{t}\left(f_{1}, f_{2}\right) \in E$ we define $\left\{u_{k}(t)\right\}$ successively as follows:

$$
u_{0}(t)=\dot{K}(t-s) f_{1}+K(t-s) f_{2}, \quad u_{k}(t)=u_{0}(t)+\Phi_{ \pm, s}\left[u_{k-1}\right](t), \quad k=1,2, \ldots
$$

The fact that $u_{0}(t) \in L_{ \pm, s}^{r} H_{p}^{1-\mu}$ follows from Lemma 2.2, and hence, we conclude from (2.12) that $u_{k}(t) \in L_{ \pm, s}^{r} H_{p}^{1-\mu}$ holds for $k \in \mathbf{N}$, successively. Moreover, since

$$
\begin{gathered}
\left\|u_{k}-u_{k-1}\right\|_{L_{ \pm, s}^{r} H_{p}^{1-\mu} \leq}\left\|\Phi_{ \pm, s}\right\|_{\mathcal{B}\left(L_{ \pm, s}^{r} H_{p}^{1-\mu}\right)}\left\|u_{k-1}-u_{k-2}\right\|_{L_{ \pm, s}^{r} H_{p}^{1-\mu}} \\
\ldots \\
\leq\left\|\Phi_{ \pm, s}\right\|_{\mathcal{B}\left(L_{ \pm, s}^{r} H_{p}^{1-\mu}\right)}^{k}\left\|u_{0}\right\|_{L_{ \pm, s}^{r} H_{p}^{1-\mu}}
\end{gathered}
$$

we see from (2.6) and (2.15) that $\left\{u_{k}(t)\right\}$ converges in $L_{ \pm, s}^{r} H_{p}^{1-\mu}$ as $k \rightarrow \infty$. Thus $u(t)$ is, in fact, the weak solution of (1.1). Moreover, we can write

$$
u(t)=u_{0}(t)+\sum_{k=1}^{\infty}\left\{u_{k}(t)-u_{k-1}(t)\right\},
$$

on account of the uniform estimates (2.9) and (2.12).

(ii) It follows from (2.2) that

$$
\begin{aligned}
\left\|\int_{S}^{t} K(t-\tau) V(\tau) u(\tau) d \tau\right\|_{H^{1}} & \leq C_{1}^{1 / 2}\|V u\|_{L_{ \pm, s}^{r^{\prime}} H_{p^{\prime}}^{\mu}} \\
& \leq C_{1}^{1 / 2}\|V\|_{L_{ \pm, s}^{v} L^{q}}\|u\|_{L_{ \pm, s}^{r} H_{p}^{1-\mu}}
\end{aligned}
$$

(2.16) together with (2.9) imply that the solution $u(t)$ of (2.9) is in $C\left(\mathbf{R}_{ \pm, s} ; H^{1}\right)$. Obviously, this $u(t)$ coincides with $U(t, s) \vec{f}$. Moreover, the estimate (2.10) easily follows from (2.12), if

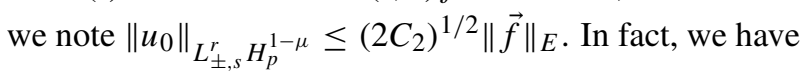

$$
\begin{aligned}
\|u\|_{L_{ \pm, s}^{r} H_{p}^{1-\mu}} & \leq\left\|u_{0}\right\|_{L_{ \pm, s}^{r} H_{p}^{1-\mu}}+\sum_{k=1}^{\infty}\left\|\Phi_{ \pm, s}\right\|_{\mathcal{B}\left(L_{ \pm, s}^{r} H_{p}^{1-\mu}\right)}^{k}\left\|u_{0}\right\|_{L_{ \pm, s}^{r} H_{p}^{1-\mu}} \\
& \leq\left\|u_{0}\right\|_{L_{ \pm, s}^{r} H_{p}^{1-\mu}}+\frac{C_{1}\|V\|_{L_{ \pm, s}^{v} L^{q}}\left\|u_{0}\right\|_{L_{ \pm, s}^{r} H_{p}^{1-\mu}}}{1-C_{1}\|V\|_{L_{ \pm, s}^{v} L^{q}}} \\
& \leq \frac{\left(2 C_{2}\right)^{1 / 2}}{1-C_{1}\|V\|_{L_{ \pm, s}^{v} L^{q}}}\|\vec{f}\|_{E},
\end{aligned}
$$

where we used (2.6) in the second step. Thus the estimate (2.11) follows from (2.10) combined with (2.17). The proof of Proposition 2.3 is finished. 


\section{Proof of Theorem 1.1}

In the following, we simply write $\vec{f}=f, \vec{f}_{0}=f_{0}$. We put $\vec{u}_{0}(t)=U_{0}(t) f_{0}$ and $\vec{u}(t)=U(t, 0) f$, and consider the inner-product $\left(\vec{u}(t), \vec{u}_{0}(t)\right)_{E}$ in $E$. Then we get, by using (1.2) and (1.3),

$$
\partial_{t}\left(\vec{u}(t), \vec{u}_{0}(t)\right)_{E}=-\frac{1}{2}\left(V u(t), u_{0 t}(t)\right),
$$

where $(\cdot, \cdot)$ stands for the usual inner-product of $L^{2}$.

Proof of Theorem 1.1 (i). Since $U(t, 0) f=U_{0}(t) U_{0}(-t) U(t, 0) f$, to verify the assertion we have only to show that $U_{0}(-t) U(t, 0)$ strongly converges in $E$ as $t \rightarrow \pm \infty$. Integrating (3.1) over $(s, t)(s<t)$, one gets

$$
\left(U_{0}(-t) U(t, 0) f-U_{0}(-s) U(s, 0) f, f_{0}\right)_{E}=-\frac{1}{2} \int_{s}^{t} \int V u u_{0 \tau} d x d \tau .
$$

By the Schwarz inequality, we have

$$
\begin{aligned}
& \left|\left(U_{0}(-t) U(t, 0) f-U_{0}(-s) U(s, 0) f, f_{0}\right)_{E}\right| \\
\leq & \frac{1}{2}\left(\int_{s}^{t} \int|V|\left|A^{\mu} u\right|^{2} d x d \tau\right)^{1 / 2}\left(\int_{s}^{t} \int|V|\left|A^{-\mu} u_{0 \tau}\right|^{2} d x d \tau\right)^{1 / 2} .
\end{aligned}
$$

By Assumption A and (2.5) we have

$$
\left(\int_{s}^{t} \int\left|V \| A^{-\mu} u_{0 \tau}\right|^{2} d x d \tau\right)^{1 / 2} \leq\left(2 C_{2}\right)^{1 / 2}\|V\|_{L_{ \pm, s}^{v} L^{q}}^{1 / 2}\left\|f_{0}\right\|_{E} .
$$

Hence it follows from (3.2)-(3.3) that

$$
\begin{aligned}
& \left|\left(U_{0}(-t) U(t, 0) f-U_{0}(-s) U(s, 0) f, f_{0}\right)_{E}\right| \\
\leq & \frac{1}{\sqrt{2}}\left|\int_{s}^{ \pm \infty} \int\right| V\left\|\left.\left.A^{\mu} u\right|^{2} d x d \tau\right|^{1 / 2} \cdot C_{2}^{1 / 2}\right\| V\left\|_{L_{ \pm, s}^{v} L^{q}}^{1 / 2}\right\| f_{0} \|_{E} .
\end{aligned}
$$

Furthermore, it follows from Assumption A and Proposition 2.3 (ii) that

$$
\begin{aligned}
& \left(\int_{s}^{t} \int\left|V \| A^{\mu} u\right|^{2} d x d \tau\right)^{1 / 2} \leq\|V\|_{L_{ \pm, s}^{v} L^{q}}^{1 / 2}\|u\|_{L_{ \pm, s}^{2 v^{\prime}} H_{2 q^{\prime}}^{\mu}} \\
& \leq\|V\|_{L_{ \pm, s}^{v} L^{q}}^{1 / 2}\|u\|_{L_{ \pm, s}^{r} H_{p}^{1-\mu}} \leq \frac{\left(2 C_{2}\right)^{1 / 2}\|V\|_{L_{ \pm, s}^{v} L^{q}}^{1 / 2}\|f\|_{E},}{1-C_{1}\|V\|_{L_{ \pm, s}^{v} L^{q}}},
\end{aligned}
$$

where we have used the relations

$$
\frac{1}{2 q^{\prime}}=\frac{1}{2}\left(1-\frac{1}{q}\right)=\frac{1}{p}, \quad \frac{1}{2 v^{\prime}}=\frac{1}{r} .
$$


The estimate (3.5) means that

$$
\left.\left.\left|\int_{s}^{ \pm \infty} \int\right| V|| A^{\mu} u\right|^{2} d x d \tau\right|^{1 / 2} \rightarrow 0, \quad(s \rightarrow \pm \infty) .
$$

Thus we conclude from (3.4) and (3.6) that $U_{0}(-t) U(t, 0)$ converges strongly in $E$ as $t \rightarrow$ $\pm \infty$.

To prove the assertion we put

$$
f_{0}^{ \pm}=s-\lim _{t \rightarrow \pm \infty} U_{0}(-t) U(t, 0) f .
$$

Then, as is expected, we conclude that

$$
\left\|U(t, 0) f-U_{0}(t) f_{0}^{ \pm}\right\|_{E}=\left\|U_{0}(t)\left\{U_{0}(-t) U(t, 0) f-f_{0}^{ \pm}\right\}\right\|_{E} \rightarrow 0
$$

as $t \rightarrow \pm \infty$.

PRoOF OF THEOREM 1.1 (ii). In this case we can apply the above argument to the operator $U_{0}(s-t) U(t, s)$ with fixed $s \in \mathbf{R}$. For this purpose, we put

$$
Z^{ \pm}(s)=s-\lim _{t \rightarrow \pm \infty} U_{0}(s-t) U(t, s) .
$$

This defines a nontrivial bounded operator on $E$ which is assured by the nondecay of the energy. We shall show that for each $0 \neq f \in E$ there exists sufficiently large $\pm s>0$ such that $Z^{ \pm}(s) U_{0}(s) f \neq 0$. Recall that $Z^{ \pm}=s-\lim _{t \rightarrow \pm \infty} U_{0}(-t) U(t, 0)$. Then since

$$
Z^{ \pm} U(0, s) U_{0}(s) f=U_{0}(-s) Z^{ \pm}(s) U_{0}(s) f
$$

$Z^{ \pm}$is verified to be a nontrivial bounded operator. Let $\vec{u}(t)=U(t, s) f$ and $\vec{u}_{0}(t)=U_{0}(t-$ s) $f_{0}$ in (3.1). Then the argument of the proof of the assertion (i) yields

$$
\begin{aligned}
& \left|\left(Z^{ \pm}(s) f, f_{0}\right)_{E}-\left(f, f_{0}\right)_{E}\right| \\
& \quad \leq\left.\left.\frac{1}{2}\left|\int_{s}^{ \pm \infty} \int\right| V\left\|\left.\left.A^{\mu} u\right|^{2} d x d \tau\right|^{1 / 2}\left|\int_{s}^{ \pm \infty} \int\right| V\right\| A^{-\mu} u_{0 \tau}\right|^{2} d x d \tau\right|^{1 / 2} \\
& \quad \leq \frac{1}{\sqrt{2}} \cdot \frac{C_{2}^{1 / 2}\|V\|_{L_{ \pm, s}^{v} L^{q}}}{1-C_{1}\|V\|_{L_{ \pm, s}^{v} L^{q}}}\|f\|_{E}\left\|u_{0 \tau}\right\|_{L_{ \pm, s}^{r} H_{p}^{-\mu}} .
\end{aligned}
$$

Let us choose here $f=f_{0}=U_{0}(s) g$ in (3.7), where $g \neq 0$, and assume that $Z^{ \pm}(s) U_{0}(s) g=$ 0 for any $\pm s>0$. Then we lead to a contradiction. It follows from Proposition 2.3 and (3.7) that

$$
\left\|U_{0}(s) g\right\|_{E} \leq \frac{1}{\sqrt{2}} \cdot \frac{C_{2}^{1 / 2}\|V\|_{L_{ \pm, s}^{v} L^{q}}}{1-C_{1}\|V\|_{L_{ \pm, s}^{v} L^{q}}}\left\|U_{0}(\tau) g\right\|_{L_{ \pm, s}^{r} H_{p}^{-\mu}} .
$$

$\left\|U_{0}(s) g\right\|_{E}$ is independent of $s$ by the energy identity $\left\|U_{0}(s) g\right\|_{E}=\|g\|_{E}>0$, whereas the right-hand side of (3.8) converges to 0 as $s \rightarrow \pm \infty$. This is a contradiction. Thus the assertion (ii) is proved. 
Proof of THEOREM 1.1 (iii). To verify the assertions (iii), we have only to show that $Z^{ \pm}(s)$ is a bijection on $E$. Assume that $g \in E$ satisfies $Z^{ \pm}(s) g=0$ or $g \perp \mathcal{R}\left(Z^{ \pm}(s)\right)$. Then putting $f=f_{0}=g$ in (3.7), we obtain

Since

$$
\|g\|_{E} \leq \frac{C_{2}\|V\|_{L_{ \pm, s}^{v} L^{q}}}{1-C_{1}\|V\|_{L_{ \pm, s}^{v} L^{q}}}\|g\|_{E} .
$$

$$
\frac{C_{2}\|V\|_{L_{ \pm s}^{v} L^{q}}}{1-C_{1}\|V\|_{L_{ \pm, s}^{v} L^{q}}}<1
$$

(3.9) implies $g=0$, and hence $Z^{ \pm}(s)$ becomes a bijection. The assertion (iii) is proved.

ACKnowledgment. The author would like to express his sincere gratitude to Professor Kiyoshi Mochizuki for many valuable suggestions on his master thesis. The author also thanks the referee for giving him some valuable comments and pointing out an error.

\section{References}

[1] P. D'AnCONA and L. FAnELli, Strichartz and smoothing estimates for dispersive equations with magnetic potentials, Comm. Partial Differential Equations 33 (2008), 1082-1112.

[ 2 ] N. HAYASHI and P. NAUMKIN, Scattering operator for nonlinear Klein-Gordon equations in higher space dimensions, J. Differential Equations 244 (2008), 188-199.

[ 3 ] J. Howland, Stationary scattering theory for time dependent Hamiltonians, Math. Ann. 207 (1974), 315335 .

[ 4 ] T. KATO, Wave operators and similarity for some non-selfadjoint operators, Math. Ann. 162 (1966), 258-279.

[5] T. KATO and K. YAJima, Some examples of smooth operators and the associated smoothing effect, Rev. in Math. Phys. 1 (1989), 481-496.

[6] H. Kitada and K. Yajima, A scattering theory for time-dependent long-range potentials, Duke Math. J. 49 (1982), 341-376.

[ 7 ] K. MochIZUKI, Scattering theory for wave equations, Kinokuniya, 1984 (in Japanese).

[ 8 ] K. MochizuKi and T. Motai, On decay-nondecay and scattering for Schrödinger equations with time dependent complex potentials, Publ. RIMS, Kyoto Univ. 43 (2007), 1183-1197.

[9] K. MochizUKI and H. NAKAZAWA, Energy decay and asymptotic behavior of solutions to the wave equation with linear dissipation, Publ. Res. Inst. Math. Sci. 32 (1996), 401-414.

[10] K. Mochizuki and H. Nakazawa, Energy decay of solutions to the wave equations with linear dissipation localized near infinity, Publ. RIMS, Kyoto Univ. 37 (2001), 441-458.

[11] S. MURAI, Scattering for Klein-Gordon equations with variable coefficients, to appear in Proc. Fac. Sch. Sci. Tokai Univ. (2009).

[12] D. R. YAFAEV, On the violation of the unitarity in time dependent potential scattering, Soviet Math. Dokl. 19 (1973), 1517-1521.

[13] K. Yajima, Existence of solutions for Schrödinger evolution equations, Comm. Math. Phys. 110 (1987), 415-426.

[14] K. YaJima, Scattering theory for Schrödinger equations with potentials periodic in time, J. Math. Soc. Japan 29 (1977), 729-743.

Present Address:

Graduate School of Science and Technology, Course of Science and Technology,

TOKAI UNIVERSITY,

HiRATSUKA, KANAGAWA, 259-1292 JAPAN.

e-mail: 8atad014@mail.tokai-u.jp 